\title{
Constitutive stabilization of hypoxia-inducible factor alpha selectively promotes the self-renewal of mesenchymal progenitors and maintains mesenchymal stromal cells in an undifferentiated state
}

\author{
In-Ho Park ${ }^{1,4}$, Kwang-Ho Kim ${ }^{1,4}$, Hyun-Kyung Choi ${ }^{1}$, Jae-Seung Shim ${ }^{1}$, Soo-Young Whang ${ }^{1}$, Sang June Hahn ${ }^{2}$, \\ Oh-Joo Kwon ${ }^{3}$ and Il-Hoan $\mathrm{Oh}^{1}$
}

With the increasing use of culture-expanded mesenchymal stromal cells (MSCs) for cell therapies, factors that regulate the cellular characteristics of MSCs have been of major interest. Oxygen concentration has been shown to influence the functions of MSCs, as well as other normal and malignant stem cells. However, the underlying mechanisms of hypoxic responses and the precise role of hypoxia-inducible factor-1 $\alpha$ (Hif- $1 \alpha$ ), the master regulatory protein of hypoxia, in MSCs remain unclear, due to the limited span of Hif-1 $\alpha$ stabilization and the complex network of hypoxic responses. In this study, to further define the significance of Hif- $1 \alpha$ in MSC function during their self-renewal and terminal differentiation, we established adult bone marrow (BM)-derived MSCs that are able to sustain high level expression of ubiquitin-resistant Hif-1 $\alpha$ during such long-term biological processes. Using this model, we show that the stabilization of Hif- $1 \alpha$ proteins exerts a selective influence on colony-forming mesenchymal progenitors promoting their self-renewal and proliferation, without affecting the proliferation of the MSC mass population. Moreover, Hif-1 $\alpha$ stabilization in MSCs led to the induction of pluripotent genes (oct-4 and klf-4) and the inhibition of their terminal differentiation into osteogenic and adipogenic lineages. These results provide insights into the previously unrecognized roles of Hif- $1 \alpha$ proteins in maintaining the primitive state of primary MSCs and on the cellular heterogeneities in hypoxic responses among MSC populations.

Experimental \& Molecular Medicine (2013) 45, e44; doi:10.1038/emm.2013.87; published online 27 September 2013

Keywords: Hif- $1 \alpha$; hypoxia; MSC; self-renewal

\section{INTRODUCTION}

Mesenchymal stromal cells (MSCs) are a non-hematopoietic adherent cell population that exhibit multi-lineage differentiation potential towards diverse types of tissues, including bone, cartilage, vessels and neurons, ${ }^{1-3}$ and secrete a wide spectrum of bioactive substances to exert a paracrine function. ${ }^{4}$ Accordingly, ex vivo expanded MSCs are currently being used in a variety of cell therapeutic trials for the regeneration of damaged cardiovascular, ${ }^{5}$ neural ${ }^{6}$ and muscular-skeletal tissues $^{7-9}$ and for facilitating hematopoietic engraftment ${ }^{10,11}$ or for suppressing grafts versus host diseases.12,13 Although MSCs can be produced from various tissues, including BM, adipose tissues or placenta, ex vivo culture-derived MSCs display several common surface phenotypes, including the expression of CD90 (Thy-1), CD 166 (SB10/ALCAM), CD73 (SH3) and CD105 (SH2, endoglin), and the absence of the hematopoietic marker (CD45), HLA-DR and co-stimulatory molecules, such as B7. However, despite these common features, significant heterogeneities have been reported for cultured MSCs in terms of their morphology, proliferation and differentiation potentials. ${ }^{14-16}$ Moreover, heterogeneities were also observed in their gene expression and differentiation potential with successive culture passages, ${ }^{17,18}$ raising the possibility that such heterogeneities could also be generated

\footnotetext{
${ }^{1}$ Catholic High-Performance Cell Therapy Center and Department of Medical Lifescience, The Catholic University of Korea, College of Medicine, Seoul, Korea; ${ }^{2}$ Department of Physiology, The Catholic University of Korea, College of Medicine, Seoul, Korea and ${ }^{3}$ Department of Biochemistry, The Catholic University of Korea, College of Medicine, Seoul, Korea

${ }^{4}$ These authors contributed equally to this work.

Correspondence: Professor I-H Oh, Catholic High-Performance Cell Therapy Center, The Catholic University of Korea, 505, Banpo-Dong, Seocho-Ku, Seoul 137-701, Korea.

E-mail: iho@catholic.ac.kr
}

Received 3 January 2013; revised 7 July 2013; accepted 10 July 2013 
during the process of ex vivo culture. Therefore, factors and underlying mechanisms involved in the regulation of the biological characteristics of ex vivo expanded MSCs have been of major interest in the field.

Recently, studies have shown that oxygen concentration can influence function in many types of stem cells. ${ }^{19}$ Such hypoxia responses are primarily mediated by signaling pathways involving HIF-1(hypoxia-inducible factor-1), ${ }^{20,21}$ the master regulatory protein of hypoxic responses, with the participation of HIF-2 or unfolded protein responses. ${ }^{22}$ Of these, Hif- $1 \alpha$ has a major role as a master regulatory protein for hypoxic responses. Hif- $1 \alpha$ is made up of two subunits; one variable (HIF-1 $\alpha$ ) and the other constant, HIF-1 $\beta$, which is also known as the aryl-hydrocarbon-receptor nuclear translocator (ARNT). Under normoxic conditions, HIF- $1 \alpha$ is hydroxylated at specific proline residues (P402, P564) by prolyl hydroxylases, which leads to the rapid degradation of HIF- $1 \alpha$ proteins through ubiquitinylation and proteosomemediated proteolysis. ${ }^{23-26}$

For MSCs, cultures under hypoxic conditions have been reported to alter the biological characteristics of MSCs. Such alterations include a higher proliferation of cells and an enhanced secretion of bioactive substances. ${ }^{27-32}$ However, despite these studies related to hypoxic responses, the role of Hif- $1 \alpha$ in the regulation of MSCs remains unclear, due to the complexity of the hypoxic responses, which can include multiple families of Hif-1-related genes ${ }^{33}$ as well as Hif- $1 \alpha$-independent pathways, such as an unfolded protein response. ${ }^{22}$ Moreover, the stability of Hif- $1 \alpha$ itself is regulated by multiple mechanisms that are dependent or independent of the hydroxylation of proline residues or pVHL pathways, ${ }^{34,35}$ making it complex to dissect the role of Hif- $1 \alpha$. Also, discrepancies in the observations on the role of hypoxia or Hif- $1 \alpha$ was reported with respect to the cell types and study models used, ${ }^{30,36-40}$ awaiting further delineation of the biological actions of Hif-1 for MSCs.

In this study, we found that the endogenous level of Hif- $1 \alpha$ or transgenic expression of wild-type (WT) Hif- $1 \alpha$ is only transiently maintained under hypoxic culture conditions, and therefore such a limited stability of Hif- $1 \alpha$ could obscure the role of Hif- $1 \alpha$ in MSCs during their prolonged biological process, such as colonization or terminal differentiation. To overcome such limitations in Hif- $1 \alpha$ stability, we established primary MSCs that were transduced with a mutant form of Hif- $1 \alpha$ that are resistant to ubiquitinylation and thereby established MSCs that stably express sustained high levels of Hif- $1 \alpha$ over prolonged culture periods. Using this model, we show that the sustained stabilization of Hif- $1 \alpha$ exerts a selective influence on colony-forming unit-fibroblasts (CFUF), a subset of mesenchymal progenitors promoting their selfrenewal and proliferation without affecting the proliferation of the MSC mass population. We also show that Hif- $1 \alpha$ stabilization drives the MSCs towards undifferentiated state while inhibiting osteogenic and adipogenic differentiation. Thus, our study reveals previously unrecognized selective role of Hif-1 to regulate self-renewal and differentiation of MSCs.

\section{MATERIALS AND METHODS}

\section{MSC culture and hypoxic conditions}

MSCs from BM aspirates obtained from a healthy donor under informed consent were prepared as previously described. ${ }^{41}$ Briefly, after Ficoll-Paque PLUS (GE Healthcare, Uppsala, Sweden) separation, BM mononuclear cells were plated in the Dulbecco's modified Eagle's medium (DMEM) containing 10\% FBS. Non-adherent cells were discarded after 1 week, and adherent populations were maintained in $10 \%$ fetal bovine serum (FBS) supplemented with DMEM at $37^{\circ} \mathrm{C}$ in a $5 \% \mathrm{CO}_{2}$ humidified incubator (Forma Scientific, Marietta, OH, USA). Culture of MSCs under hypoxic conditions was performed by placing cells in a $\mathrm{CO}_{2}$ water-jacketed incubator (Thermo Forma model 3110) or alternatively by using hypoxia chamber (Galaxy 48R, Eppendorf, Hamburg, Germany) with oxygen sensor attached for monitoring of $\mathrm{O}_{2}$ concentration in the chamber.

The study was approved by the Institutional Review Board of The Catholic University of Korea.

\section{Vector construction, virus production and transduction to MSCs}

The parental plasmid encoding WT HIF-1 $\alpha$ (Figure 1a) and HIF- $1 \alpha$ double-mutant (Hif- $1 \alpha$ Flag-P402A/P564A, abbreviated as PA, Figure 1a) was provided by Professor KW Kim (Neurovascular Coordination Research Center, College of Pharmacy, Seoul National University, Seoul, Korea). For the preparation of engineered MSCs

a

\begin{tabular}{|c|c|c|c|c|c|}
\hline \multirow[b]{2}{*}{ HIF-1 $\alpha-W T$} & & & P402 & $P 564$ & \multirow[b]{2}{*}{ TAD } \\
\hline & bHLH & PAS & & TAD & \\
\hline & & & \multicolumn{2}{|c|}{ ODD } & \\
\hline & & & A402 & A564 & \\
\hline HIF-1 $\alpha-P A$ & bHLH & PAS & & TAD & TAD \\
\hline
\end{tabular}

b
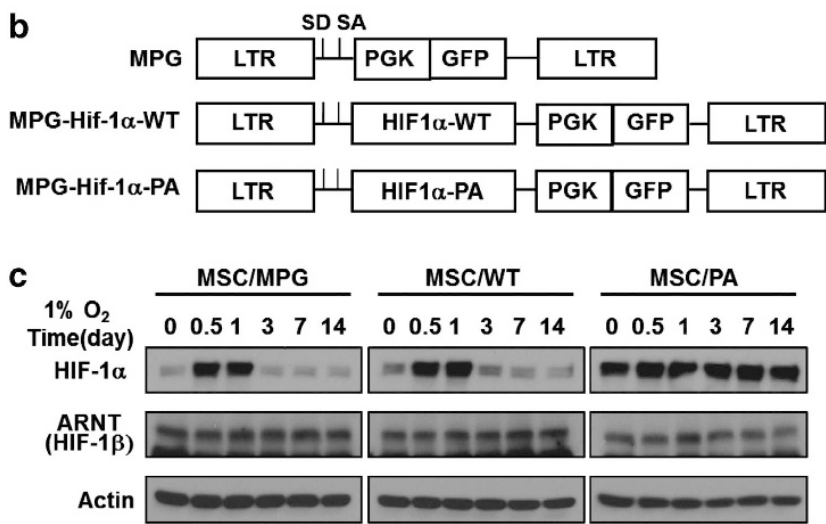

Figure 1 Kinetics of protein stabilization for various forms of Hif- $1 \alpha$. (a) Schematic structure of wild-type (WT) and ubiquitinresistant form of Hif-1 $\alpha$ (PA). bHLH; basic helix-loop-helix domain, PAS; Per/Arnt/Sim, ODD; oxygen-dependent degradation domain, TAD; transactivation domain. The proline residues (P402, p564) in ODD were substituted for Ala residues in the Hif- $1 \alpha-P A$ mutant. (b) Structures of retroviral vectors WT or PA mutant form of Hif- $1 \alpha$ along with green fluorescent protein (GFP). (c) Comparisons for kinetics of Hif- $1 \alpha$ and Hif-1 $\beta$ (ARNT) protein upon exposure to hypoxia $\left(\begin{array}{ll}1 \% & \mathrm{O}_{2}\end{array}\right)$. Shown are representative western blots for each indicated protein in MSCs during incubation under hypoxic conditions. 
overexpressing HIF- $1 \alpha$ WT or PA mutant, each HIF- $1 \alpha$ cDNAs were first cloned into the murine stem cell virus vector (MSCV) expressing green fluorescent protein (GFP) under the phosphoglycerate kinase (PGK) promoter (MSCV-PGK-GFP, abbreviated as MPG, Figure $1 \mathrm{~b})^{42}$ and retroviral particles were produced by the cotransfection of $293 \mathrm{~T}$ cells with each retroviral vector (MPG, MPG-WT or MPG-PA) plus plasmids containing gag-pol, vesicular stomatitis virus-glycoprotein and gibbon ape leukemia virus envelope as previously described. ${ }^{43}$ The supernatants were then collected, concentrated by ultracentrifugation and used to infect MSCs. After transduction, transduced MSCs were purified by sorting for $\mathrm{GFP}^{+}$cells.

\section{Proliferation assay}

To compare expansion under normoxic and hypoxic culture conditions, MSCs that had been maintained under identical conditions (DMEM with 10\% FBS) were transferred and subcultured in normoxic $\left(21 \% \mathrm{O}_{2}\right)$ and hypoxic $\left(1 \% \mathrm{O}_{2}\right)$ culture conditions for at least two passages before analysis. Doubling times were calculated as $t / n$, where $t$ is the duration of the culture and $n$ is the number of population doublings calculated using the formula $n=\log (\mathrm{NH}-\mathrm{NI})$ / $\log 2$ (where NI is the number of cells originally plated and $\mathrm{NH}$ the number of cells harvested at the time of counting). Bromodeoxy uridine (BrdU ) incorporation of MSCs were measured by incubating the cells with $10 \mu \mathrm{M}$ BrdU for $2 \mathrm{~h}$ in the medium, followed by staining with allophycocyanin-conjugated antibody against BrdU (BD Bioscience, San Jose, CA, USA) along with propidium iodide for flow cytometry.

\section{Colony-formation assay}

For colony formation (CFU-F) in in vitro cultured MSCs, MSCs were pre-incubated under normoxic or hypoxic conditions for 7 days, then plated at a density of 1000 cells per $100 \mathrm{~mm}$ dish, and after incubation for 14 days under normoxic or hypoxic conditions, the number of colonies containing $>50$ cells was counted after staining with crystal violet (Sigma, St Louis, MO, USA) in methanol.

\section{Reverse transcriptase-PCR}

Total RNA was isolated using the TRIzol reagent (Invitrogen, Carlsbad, CA, USA), and cDNAs were synthesized according to the manufacturer's instructions (SuperScript II, Invitrogen). GAPDH was used for normalization. Primers used are listed in Supplementary Table 1. PCR parameters were $95^{\circ} \mathrm{C}$ for $5 \mathrm{~min}$ followed by 30 cycles of $95^{\circ} \mathrm{C}$ for $30 \mathrm{~s}, 55-60{ }^{\circ} \mathrm{C}$ for $30 \mathrm{~s}$ and $72{ }^{\circ} \mathrm{C}$ for $30 \mathrm{~s}$.

\section{Flow cytometry analysis}

Flow cytometry analysis of MSC surface markers was performed as described in a previous study. ${ }^{41}$ Briefly, cells were stained with monoclonal antibodies, anti-human CD34-PE, CD73-PE, CD146-PE, CD271-PE and CD140b (PDGFR- $\beta$ ) (BD Pharmingen, San Diego, CA, USA) and analyzed using FACSCalibur (Becton Dickinson, San Jose, CA, USA) and CellQuest software (BD Bioscience).

\section{Osteogenic and adipogenic differentiation}

Osteogenic differentiation was induced by DMEM supplemented with $10 \%$ FBS, $10 \mathrm{~nm}$ dexamethasone, $0.2 \mathrm{~mm}$ ascorbic acid and $10 \mathrm{~mm}$ $\beta$-glycerophosphate as described. ${ }^{44}$ After 28 days, the mineralization of the extracellular matrix was determined by Alizarin Red S staining, which was quantified by dye elution using cetylpyridinium chloride (Sigma) and measurement of the absorbance at $550 \mathrm{~nm}$.
For adipogenic differentiation, confluent cells were incubated in DMEM containing 10\% FBS, $1 \mu \mathrm{M}$ dexamethasone, $0.5 \mathrm{~mm}$ isobutyl1-methylxanthine, $100 \mu \mathrm{m}$ indomethacin and $10 \mu \mathrm{g} \mathrm{ml}^{-1}$ insulin. ${ }^{42}$ After 14 days, cells were fixed with $4 \%$ formaldehyde and stained with Oil Red $\mathrm{O}$ to visualize lipid droplets. The number of differentiated adipocytes was subsequently determined by dye extraction using $4 \%$ Nonidet P40 in isopropyl alcohol followed by spectrophotometry at $520 \mathrm{~nm}$.

\section{Neural induction}

Subconfluent cultures of MSCs were maintained under normoxic or hypoxic conditions for $48 \mathrm{~h}$. To initiate neural differentiation, the media was removed, and the cells were washed with phosphatebuffered saline and transferred to neural induction media composed of DMEM/2\% dimethyl sulfoxide/25 mM KCl/100 mM BHA/100 mM forskoin/2 mM valproic acid/10 $\mathrm{ng} \mathrm{ml}^{-1}$ basic fibroblast growth factor/ N2 supplement for $24 \mathrm{~h}^{45}$

\section{Electrophysiology}

Whole-cell patch-clamp recordings were performed with an Axopatch 700B patch-clamp amplifier and pClamp 10.0 software (Molecular Devices, Sunnyvale, CA, USA) at room temperature $\left(22-24^{\circ} \mathrm{C}\right)$ as described previously. ${ }^{46}$ The tip resistance of the patch pipettes was between 2 and $4 \mathrm{M} \Omega$ when filled with the internal solution. The extracellular bath solution contained (in $\mathrm{mm}$ ) $140 \mathrm{NaCl}, 3 \mathrm{KCl}$, $1 \mathrm{CaCl}_{2}, 1 \mathrm{MgCl}_{2}, 20 \mathrm{HEPES}$ and 10 glucose and was adjusted to $\mathrm{pH}$ 7.3 using $\mathrm{NaOH}$. The intracellular pipette solution contained (in mм) $140 \mathrm{CsCl}, 10 \mathrm{NaCl} 1 \mathrm{MgCl}_{2}, 10$ HEPES and 1 EGTA and was adjusted to $\mathrm{pH} 7.3$ using $\mathrm{CsOH}$. Linear leakage and capacitance currents were corrected using P/4 subtraction method. Measured osmolarity of the extracellular bath solution was 300-340 mOsm.

\section{Immunoblot analysis}

MSCs were washed with phosphate-buffered saline and lysed in 2X Laemmli sample buffer ( $120 \mathrm{~mm}$ Tris- $\mathrm{HCl}, \mathrm{pH} 6.8,20 \%$ glycerol, $4 \%$ sodium dodecyl sulfate, $28.8 \mathrm{~mm}$ 2-mercaptoethanol, $0.01 \%$ bromophenol blue). Cell lysates were boiled for $5 \mathrm{~min}$. and cleared by centrifugation at $18000 \mathrm{~g}$ for $15 \mathrm{~min}$. Forty micrograms of protein, as determined using an RCDC protein assay kit (Bio-Rad, Hercules, CA, USA), was electrophoresed in acrylamide-sodium dodecyl sulfate denaturing gels. Gels were transferred to Immobilon (Millipore, Bedford, MA, USA) and probed with antibodies as recommended by the suppliers. Antibody against Hif- $1 \alpha$ was purchased from Cayman Chemical (Ann Arbor, MI, USA), antibody against NSE from Abcam (Cambridge, UK), antibodies against TAZ from Santa Cruz Biotech (Santa Cruz, CA, USA), antibodies against ARNT from Upstate (Lake Placid, NY, USA), antibody against pan-neuronal neurofilament from Covance (Emeryville, CA, USA), antibody against tubulin b-III from Chemicon (Lake Placid), antibody against p21 (Cell Signaling, Danvers, MA, USA), antibody against p27 (Cell Signaling) and antibody against actin (Millipore). Detection of specific protein was performed using an enhanced chemiluminescence system.

\section{Statistical analysis}

All experiments were performed a minimum of three times. Data are expressed as the mean \pm s.e. and were analyzed by the Student's $t$-test. $P$-value $<0.05$ was considered to be statistically significant. 


\section{RESULTS}

Establishment of human MSCs expressing sustained high levels of Hif- $1 \alpha$ proteins

To explore the role of Hif- $1 \alpha$ during the long-term period of biological process of MSCs, we first compared the kinetics of protein accumulation for various forms of Hif- $1 \alpha$ proteins. For this, retroviral vectors (MPG) expressing GFPs along with WT Hif- $1 \alpha$ (MPG-Hif- $1 \alpha$-WT) or a mutant form of Hif- $1 \alpha$ (MPG-Hif-1 $\alpha$-PA) in which the proline residues (p402 and p564) in oxygen-dependent degradation domain were mutated, to make the protein resistant to ubiquitin-mediated proteolysis $^{33}$ (Figures 1a and b). Primary human BM-derived MSCs were then transduced with each of the retroviral vectors, sorted for transduced (GFP + ) cells, and the protein levels of Hif- $1 \alpha$ in these MSCs were examined under normoxic and hypoxic conditions. As shown in Figure 1c, the endogenous levels of the Hif- $1 \alpha$ protein in control MSCs (MSC/MPG) rapidly increased within $12 \mathrm{~h}$ after exposure to hypoxia but were maintained only for a transient period reaching basal levels after 3 days. Similarly, MSCs transduced with WT Hif- $1 \alpha$ (MSC/WT) exhibited a higher level of protein accumulation under hypoxia but also exhibited a transient accumulation of WT proteins. In contrast, MSCs that had been transduced with the mutant form HIF-1 $\alpha$ (MSC/PA) exhibited a sustained high level accumulation of the protein under normoxia as well as under hypoxic conditions throughout the period of observation (up to 14 days) (Figure 1c). Similar differences in protein stability between the MSCs transduced with WT and Hif- $1 \alpha$ PA mutant were also observed under 5\% hypoxic conditions (Supplementary Figure 1A). However, no significant change in ARNT, the $\beta$-subunit of Hif-1, was detected in any of the conditions examined (Figure 1c). In addition, the MSCs that had been transduced with Hif-1 $\alpha$-PA exhibited induction of their known target genes such as vascular endothelial growth factor and Glut- $1,{ }^{33}$ confirming their transactivation function (Supplementary Figure 1B). These results indicate that MSCs that were transduced with Hif- $1 \alpha-\mathrm{PA}$, but not WT Hif- $1 \alpha$, can serve as a model for a sustained high level accumulation of Hif- $1 \alpha$ proteins to dissect their role in MSCs during the longterm biological process of MSCs.

\section{Hif- $1 \alpha$ selectively promotes self-renewal and the proliferation of mesenchymal colony-forming cells but not the mass population of MSCs}

Using this study model, we first examined the effects of hypoxia on the proliferative activity of the mass population of MSCs by analyzing changes in their cell cycling activities. As shown, human MSCs exhibited slightly higher progression into S-phase as evidenced by higher incorporation of BrdU (Figure 2a), which was associated with decreased protein levels of p21 or p27 (Figure 2b). In consistent with the findings, MSCs transduced with the control vector (MSC/MPG) exhibited a moderately higher proliferation and shorter doubling times under hypoxic $\left(\begin{array}{ll}1 \% & \mathrm{O}_{2}\end{array}\right)$ culture conditions compared with cultures under normoxic conditions. (Figures $2 \mathrm{c}$ and $\mathrm{d}$ ). Similar increases of proliferative activity under hypoxic condition were also observed for MSCs transduced with Hif-1 $\alpha$-PA (MSC/ PA). However, MSCs transduced with Hif- $1 \alpha-\mathrm{PA}$ did not exhibit any higher proliferative activity than control MSCs under normoxic conditions as well as under hypoxic conditions. Moreover, the extent of increment in growth rate in response to hypoxia for MSC/PA was not higher than that for MSC/MPG (Figures 2c and d). A similar increment in MSC proliferation by hypoxia but a lack of difference in growth between the MSC/MIG and MSC/Hif-1 $\alpha$-PA was also observed under $5 \%$ hypoxic culture conditions (Supplementary Figure 2). These results show that, while hypoxia causes moderate increase in the proliferation of MSCs, Hif- $1 \alpha$ stabilization neither have a role in promoting the proliferation of MSC mass populations nor in the magnitude of their response to hypoxia.

However, considering the notion that MSCs exhibit extensive heterogeneities among their subpopulations, ${ }^{14-16}$ we hypothesized that heterogeneities could conceivably exist in their response to Hif- $1 \alpha$ stabilization for each distinct subpopulation of MSCs. To explore this possibility, we examined the effects of Hif- $1 \alpha$ stabilization on the self-renewal of mesenchymal progenitors, a primitive population of MSCs characterized by their ability to form CFU-F. For this, MSCs were culture expanded under hypoxic or normoxic conditions for 7 days and examined for their frequency of CFU-F by plating equivalent numbers of expanded cells under identical normoxic condition (illustrated in Figure 3a, upper panel). As shown in Figure 3a, (middle and low panels), the numbers of CFU-F produced from MSC/MGP were increased sharply under hypoxic conditions compared with normoxic conditions, indicating a higher self-renewal of mesenchymal colony-forming progenitors under hypoxic culture conditions. Notably, MSCs transduced with Hif- $1 \alpha$-PA exhibited a similar increase in the frequency of CFU-F, even under the normoxic conditions, to the levels comparable to those achieved by hypoxic culture of MSC/MPG. These findings suggest that stabilized Hif- $1 \alpha$ has a direct role in promoting the self-renewal of mesenchymal progenitors mediating the effects of hypoxia for the subsets of MSCs.

Having observed the effects on self-renewal of progenitors during expansion cultures, we next examined the effects of Hif- $1 \alpha$ and hypoxia on colony formation from given numbers of mesenchymal progenitors by plating equivalent numbers of MSCs for colonization under normoxic or hypoxic conditions (illustrated in Figure 3b, upper panel). As shown in the Figures, the colonies produced from Hif-1 $\alpha$-PA-MSCs were markedly larger than those from control MSCs both under normoxic and hypoxic condition (Figure 4b, middle panel), indicating that Hif- $1 \alpha$ stabilization caused a higher proliferative activity of colony-forming cells during the colonization process. Interestingly, the numbers of colonies from MSC/PA were significantly higher than those from MSC/MPG under normoxic conditions (Figure 3b, lower panel). However, considering the fact that identical numbers of colony-forming cells had been plated at the initiation of colonization, these results suggest that an increase in the size of colonies can be 
a

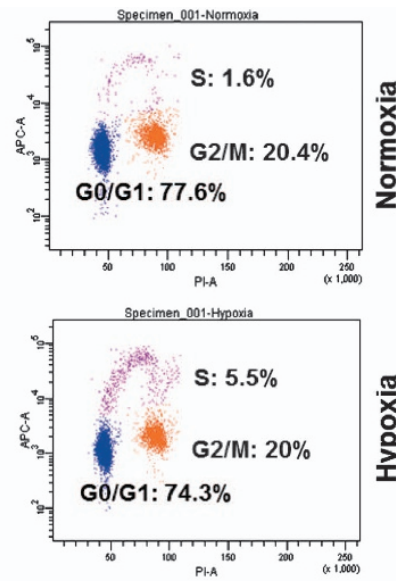

c

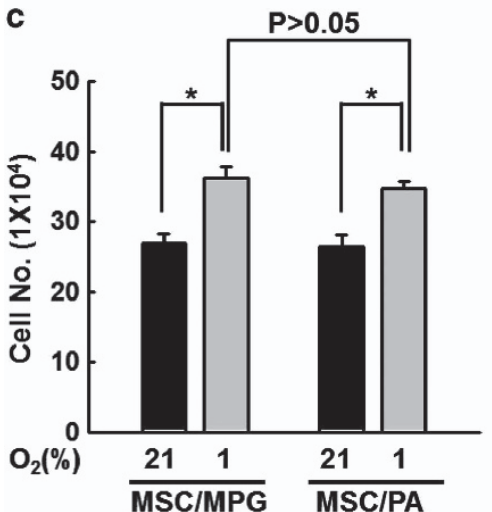

b

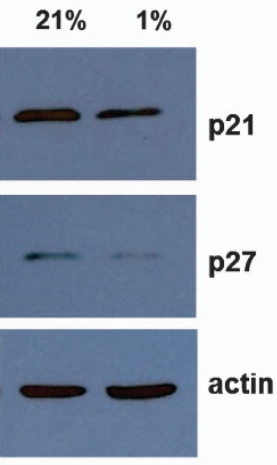

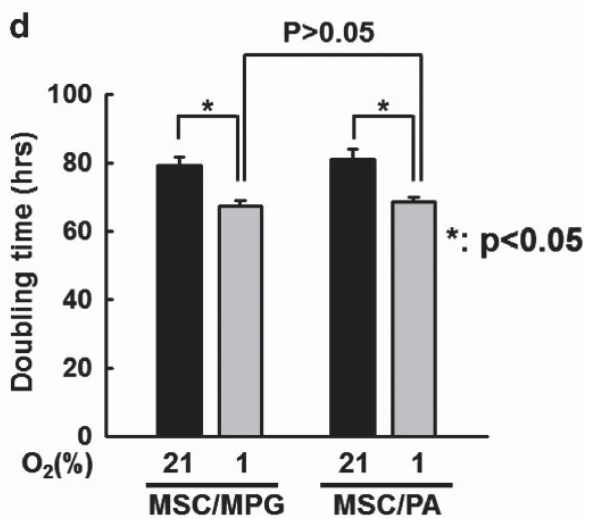

Figure 2 Effects of hypoxia and Hif- $1 \alpha$ stabilization on the growth of MSCs in mass population. (a) BrdU incorporation analysis of MSCs under normoxic or hypoxic conditions. Cells were added with $10 \mu \mathrm{m}$ of BrdU in the culture medium for $2 \mathrm{~h}$. MSCs were then stained with antibody against BrdU and propidium iodide (PI) for flow cytometric analysis. Shown are the representative plots and percentage of cells in each cell cycle fraction. (b) Effects of hypoxia on cell cycle inhibitory proteins in MSCs. MSCs were cultured under 21 or $1 \% \mathrm{O}_{2}$ for 2 days and analyzed for protein levels of each indicated gene by using antibody specific to p21 or p27. (c) MSCs were maintained under hypoxic $\left(1 \% \mathrm{O}_{2}\right)$ or normoxic $\left(21 \% \mathrm{O}_{2}\right)$ for at least two passages and plated in the dish $\left(5 \times 10^{4} / 100 \mathrm{~mm}\right.$ dish density) at equal density. Eight days after plating, the cell numbers were counted. Shown are the mean \pm s.e.m. values from three experiments. (d) Doubling times were calculated as $t / n$, where $t$ is the duration of culture and $n$ is the number of population doublings calculated by using the formula $n=\log (\mathrm{NH}-\mathrm{NI}) / \log 2$ (where $\mathrm{NI}$ is the number of cells originally plated and $\mathrm{NH}$ the number of cells harvested at the time of counting). Shown are the mean \pm s.e.m. values from three experiments. APC, allophycocyanin.

attributed to the higher read-outs of colony number secondary to the higher proliferation of progenitors during the colonization process. Collectively, these results show that Hif- $1 \alpha$ stabilization could promote the proliferative activity of mesenchymal progenitors during the colonization process as well as the self-renewal of the progenitors during expansion culture.

To determine whether this increase in colony numbers and proliferative activity is associated with concomitant molecular changes, we next examined the expression of oct-4, the induction of which were previously shown to be correlated with the primitive phenotype and higher proliferation. ${ }^{47,48}$ Consistent with the previous findings, the control MSCs (MSC/MPG) cultured under hypoxic conditions exhibited the induction of oct- 4 after exposure to hypoxia for periods lasting up to 7 days (Figure 4). Interestingly, MSCs that had been transduced with Hif- $1 \alpha$-PA exhibited significantly higher level expression of oct- 4 and klf- 4 than control MSCs even during normoxic culture conditions, indicating that the stabilization of Hif- $1 \alpha$ can induce the expression of primitive pluripotent genes in MSCs.

Together, these findings show that the stabilization of Hif- $1 \alpha$ exerts an influence on MSCs driving the cells towards a more primitive molecular state and promotes the self-renewal and proliferative potential of mesenchymal progenitor populations.

\section{Stabilization of Hif- $1 \alpha$ inhibits both adipogenic and} osteogenic differentiation but drives MSCs towards neuronlike cells

Having observed the effects of Hif- $1 \alpha$ on self-renewal and proliferation, we next examined the effects of Hif- $1 \alpha$ on the differentiation potential of MSCs towards osteogenic or adipogenic differentiation. As shown in Figure 5a, osteogenic differentiation of control MSCs (MSC/MPG) were markedly decreased under hypoxic culture conditions, as evidenced by their morphology and quantitative measurements of calcium precipitation (Figures $5 \mathrm{a}$ and $\mathrm{b}$ ). Similarly, the induction of 


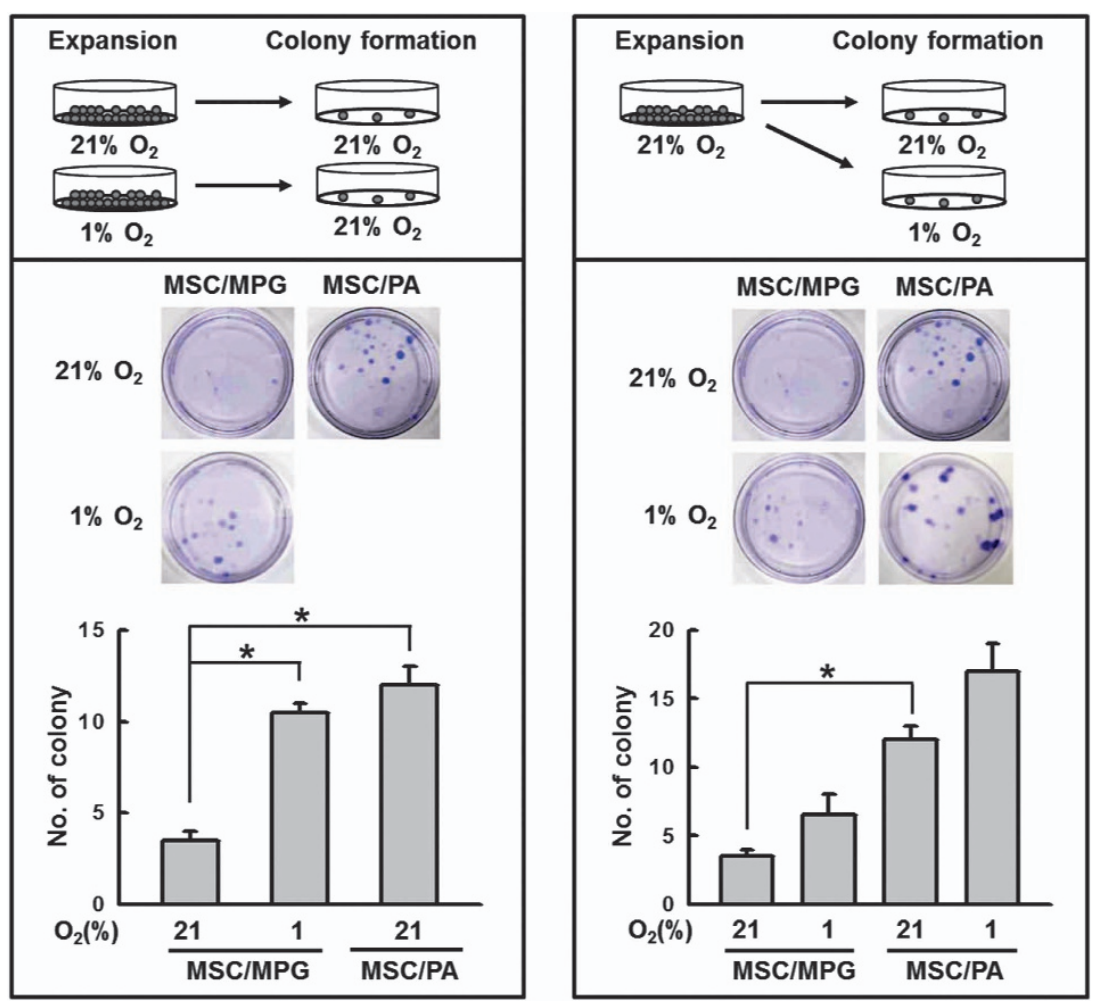

Figure 3 (a) Effects of hypoxia and Hif- $1 \alpha$ stabilization during expansion culture on the self-renewal of colony-forming mesenchymal progenitor cells. (Upper) Illustration of the experimental scheme. Control (MSC/MPG) or Hif-1 $\alpha$-PA-transduced MSCs (MSC/PA) were maintained under normoxic $(21 \%)$ or hypoxic $(1 \%)$ conditions for two passages (7 days), equal numbers of each group of MSCs were then plated for colonization under normoxia. (Middle) Representative morphology of colony formation for each group of MSCs. (Lower) Number of CFU-F formed from plating 1000 cells of each group of MSCs in the 100-mm dish. Shown are the mean \pm s.e.m. values from four independent experiments. ${ }^{*} P<0.05$. (b) Effects of hypoxia and Hif- $1 \alpha$ stabilization on the colonization process of MSCs. (Upper) Illustration of the experimental scheme. Control (MSC/MPG) or Hif- $1 \alpha$-PA-transduced MSCs (MSC/PA) were maintained under normoxic $(21 \%)$ or hypoxic ( $1 \%)$ conditions for two passages ( 7 days), then equal number (1000/well) of each group of MSCs were plated under normoxic $(21 \%)$ or hypoxic (1\%) conditions for colonization of mesenchymal progenitors. (Middle) Representative morphology of colony formation for each group of MSCs. (Lower) Number of CFU-F formed from plating 1000 cells of each group of MSCs in a100-mm dish. Shown are the mean \pm s.e.m. values from four independent experiments. ${ }^{*} P<0.05$.

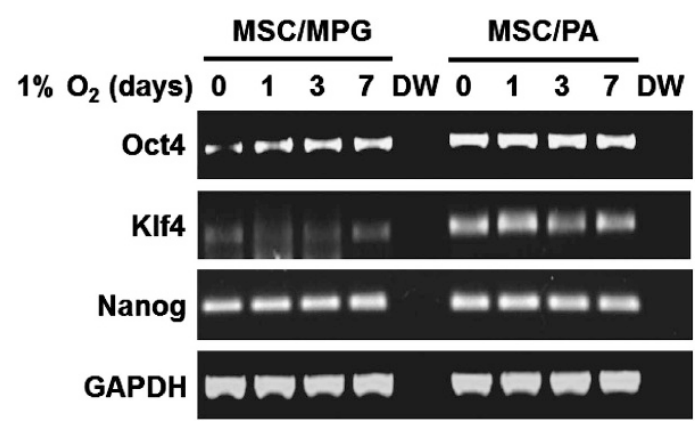

Figure 4 Effects of Hif- $1 \alpha$ stabilization on the expression of pluripotent genes in MSC. Control (MSC/MPG) or Hif- $1 \alpha$-PA-transduced MSCs (MSC/PA) were exposed to $1 \%$ hypoxia and expression of each indicated gene was analyzed by reverse transcriptase-PCR. GAPDH, glyceraldehyde 3-phosphate dehydrogenase.

osteogenic genes such as osteocalcin and osteopontin during differentiation, which was observed in MSC/MPG under normoxic conditions, was not observed under conditions of hypoxic differentiation (Figure 5c). Notably, MSCs that had been transduced with Hif- $1 \alpha$-PA (MSC/PA) exhibited a similar inhibition in osteogenic differentiation and osteogenic gene induction under normoxic conditions to comparable levels as for MSC/MPG under hypoxic culture (Figures $5 a-c$ ).

Moreover, the transcriptional coactivator regulating osteogenic differentiation of MSCs, TAZ, ${ }^{49}$ was upregulated during differentiation under normoxia, but this stabilization of TAZ was profoundly suppressed in the case of MSC/MPG under hypoxic conditions as well as in the MSC/PA under normoxic differentiation conditions (Figure 5d). These results show that the stabilization of Hif- $1 \alpha$ directly has multiple roles to inhibit the osteogenic differentiation of MSCs.

We next examined the effects of Hif- $1 \alpha$ stabilization on the adipogenic differentiation of MSCs. As shown in Figure 6, the adipogenic differentiation of MSC/MPG was also inhibited under hypoxic conditions, albeit to a moderate extent, and the expression of Hif- $1 \alpha$-PA caused a similar degree of inhibition, as evidenced by cell morphology and a quantitative decrease of fat droplets (Figures 6a and b). The decrease in adipogenic differentiation was also associated with lower expression levels 
a

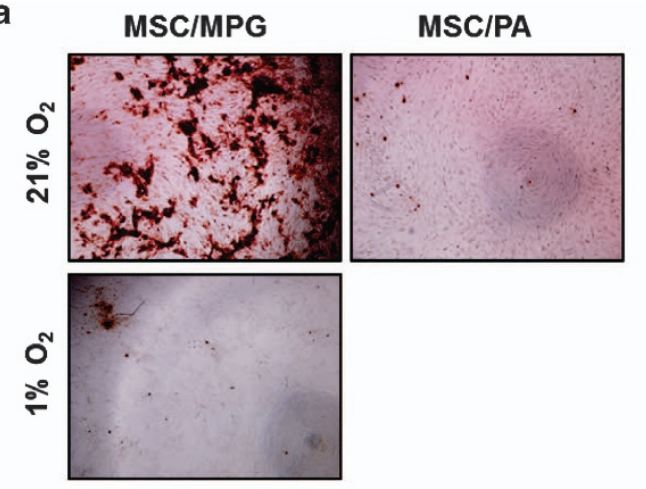

c

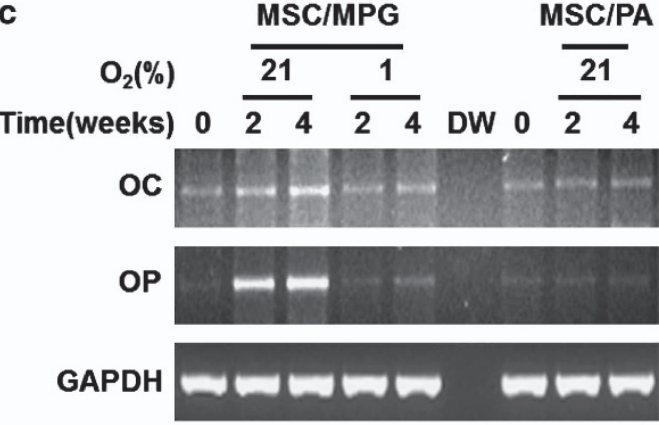

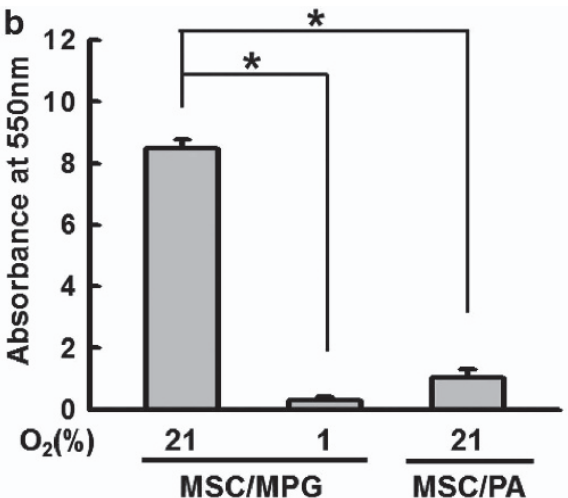

d $\mathrm{O}_{2}(\%)$ MSC/MPG MSC/PA

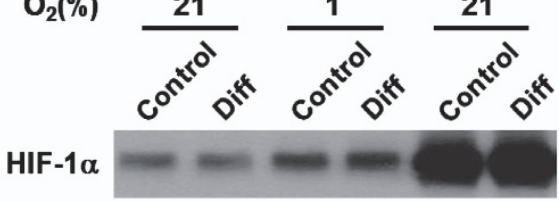

TAZ

Actin

Figure 5 Effects of hypoxia and Hif- $1 \alpha$ stabilization on the osteogenic differentiation of MSCs. (a, b) Control (MSC/MPG) or Hif- $1 \alpha-P A-$ transduced MSCs (MSC/PA) were subjected to osteogenic differentiation by chemically defined medium under normoxic or hypoxic (1\%) conditions. At 14 days after differentiation, the cells were examined by Alizarin Red S staining and quantification of mineralized nodules as determined by absorbance at $550 \mathrm{~nm}$ after dye elution. Shown are representative morphology and mean \pm s.e.m. values from four independent experiments. ${ }^{*} P<0.05$. (c) Expression of osteogenic gene expression during differentiation was analyzed by reverse transcriptase-PCR. (d) Stabilization of TAZ during osteogenic differentiation of each group of MSCs were compared with each group of MSCs during normoxia and hypoxia $\left(1 \% \mathrm{O}_{2}\right)$. GAPDH, glyceraldehyde 3-phosphate dehydrogenase; OC, osteocalcin; OP, osteopontin.
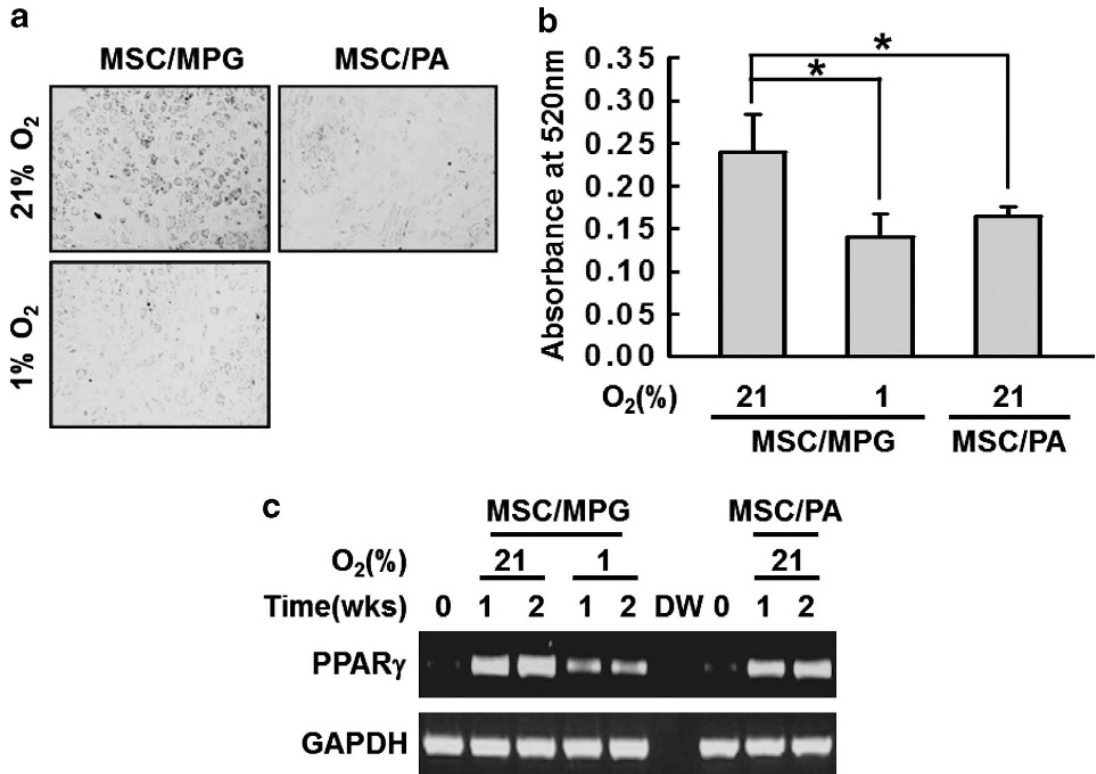

Figure 6 Effects of hypoxia and Hif- $1 \alpha$ on the adipogenic differentiation of MSCs. Each group of MSCs were subjected to adipogenic differentiation by chemically defined media under normoxic or hypoxic conditions. (a, b) After 7 days in their induction media, MSCs were stained with Oil-Red $\mathrm{O}$ and lipid accumulation was quantified by dye extraction and spectrophotometry at $502 \mathrm{~nm}$. Shown are the mean \pm s.e.m. values from four independent experiments. ${ }^{*} P<0.05$. (c) Expression of peroxisome proliferator-activated receptor- $\gamma($ PPAR $\gamma)$ during adipogenic differentiation was analyzed by reverse transcriptase-PCR. GAPDH, glyceraldehyde 3-phosphate dehydrogenase. 

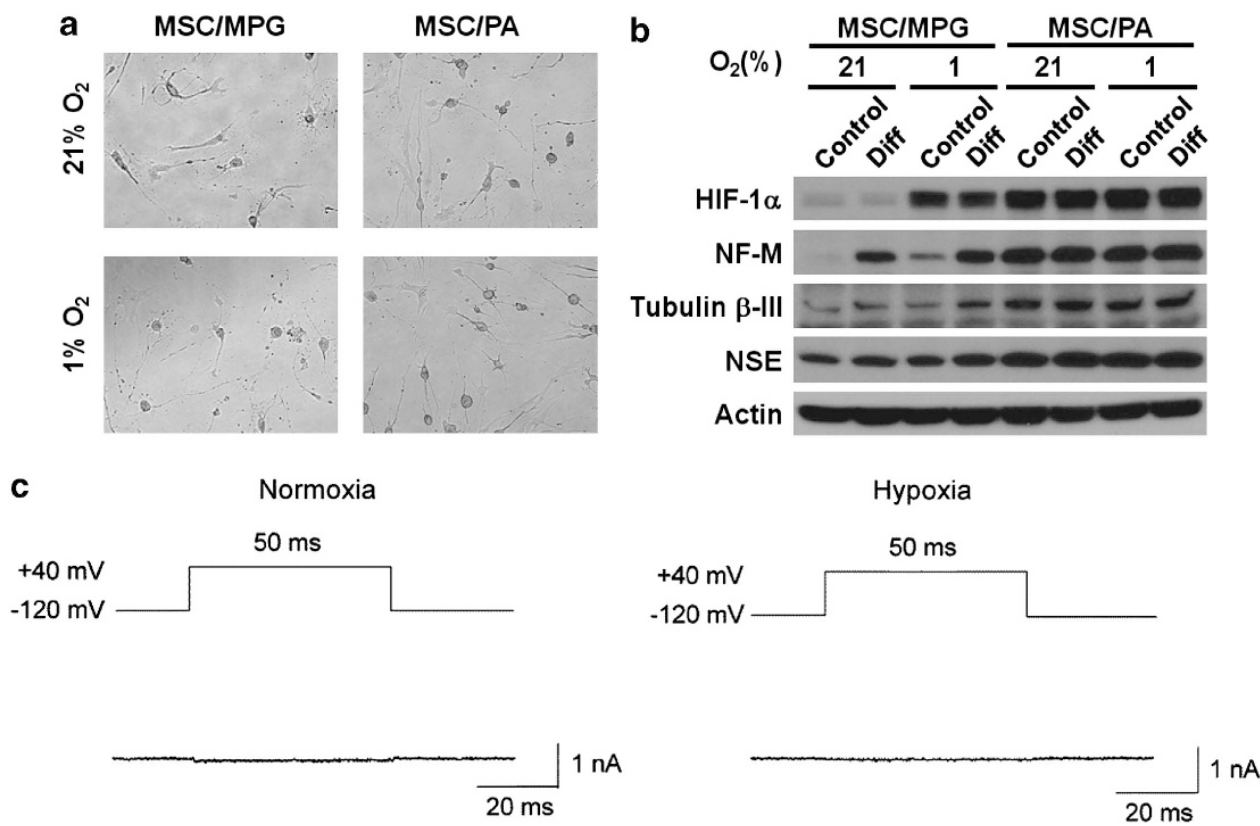

Figure 7 Effects of hypoxia and Hif-1 $\alpha$ on the neuronal differentiation of MSCs. Each indicated group of MSCs were subjected to neuronal differentiation by chemically defined media under normoxic or hypoxic conditions. Twenty-four hours after induction under normoxic or hypoxic conditions, MSCs were examined by (a) morphology and the expression of each indicated neuron-specific genes was determined by (b) western blot analysis. (c) Original current recordings in normoxic or hypoxic cells. Whole-cell current was elicited by a 50-ms depolarizing pulse to $+40 \mathrm{mV}$ from a holding potential of $-120 \mathrm{mV}$ at 10 -s intervals.

of peroxisome proliferator-activated receptor- $\gamma$ during the differentiation of MSC/MPG under hypoxic conditions and of MSC/PA under normoxic conditions (Figure 6c). These results show that, unlike a previous study suggesting Hif- $1 \alpha-$ independent inhibition of adipogenic differentiation under hypoxia, ${ }^{40}$ constitutive stabilization of Hif- $1 \alpha$ can also negatively regulate the terminal differentiation of MSCs into an adipogenic lineage.

On the contrary, when the MSCs were induced to differentiate into neuronal lineages, the neuron-like morphology was increased in MSC/MPG under hypoxic conditions, and similar change was observed in Hif-1 $\alpha$-PA-transduced MSCs under normoxic conditions (Figure 7a). Moreover, while neuron-specific genes such as NF-M (neurofilamentmedium), $\beta$-tubulin-1ll or NSE (neuron-specific enolase) were induced during neuronal differentiation, the levels of these genes were markedly upregulated in Hif- $1 \alpha$-PA-transduced MSCs, even before neuronal differentiation was induced (Figure $7 \mathrm{~b}$ ). To examine the neuronal excitation function of those cells exhibiting neuron-like morphology, we performed a electrophysiological recording for inward sodium currents in MSCs differentiated under normoxic or hypoxic cells. The cell capacitances were $113.5 \pm 26.6 \mathrm{pF}(n=5)$ and $119.7 \pm 34.6 \mathrm{pF}$ $(n=5)$ for cells differentiated under normoxic or hypoxic conditions, respectively, indicating no significant difference between the two. When whole-cell current was elicited by a 50 -ms depolarizing pulse to $+40 \mathrm{mV}$, no appreciable endogenous inward currents were detected in these cells $(n=10$; Figure $7 \mathrm{c}$ ), suggesting that constitutive stabilization of Hif- $1 \alpha$ might prime the MSCs towards neuron-like cells but not to the stage of functional neuron.

Collectively, these results show that Hif- $1 \alpha$ has a pleiotrophic role in regulating the cell fate of MSCs, promoting an undifferentiated state of MSCs and the self-renewal of progenitors while driving the cells towards a neuron-like state.

\section{DISCUSSION}

The MSCs obtained by ex vivo expansion have been frequently used in a wide diversity of cell therapeutic trials, but they exhibit significant heterogeneities in their biological characteristics, such as morphology, self-renewal, proliferation, differentiation potentials and their own gene expression patterns. ${ }^{14-18}$ Thus, culture conditions and cellular signals that can influence the potential therapeutic outcomes of MSCs have been of great interest.

The oxygen concentration in the culture is a crucial factor that can significantly influence the proliferation and differentiation potentials of MSCs. ${ }^{30,36,39,40}$ Accordingly, the role of Hif- $1 \alpha$ as a key mediator of the hypoxic response has been extensively studied with regard to the regulation and biochemical functions in MSCs ${ }^{20,21}$ However, despite these interest, studies on the precise role of Hif- $1 \alpha$ during the hypoxic response of MSCs are complicated by the fact that Hif- $1 \alpha$-independent pathways such as unfolded protein response are involved in hypoxic responses. ${ }^{22}$ Moreover, prolyl hydroxylase, an enzyme that can influence the stability of Hif- $1 \alpha$ proteins, was also shown to be induced under hypoxic conditions, ${ }^{38}$ leading to a limited stability of Hif- $1 \alpha$ during hypoxia. 
In this study, using an ubiquitin-resistant form of Hif- $1 \alpha$ as a model, we analyzed the impact of sustained Hif- $1 \alpha$ stabilization on MSCs during colony formation or terminal differentiation, in an attempt to precisely define the biological impact of Hif- $1 \alpha$ on various biological functions of MSCs.

We found that Hif- $1 \alpha$ proteins significantly enhanced the frequency and proliferative activity of colony-forming mesenchymal progenitors as evidenced by the increased numbers and sizes of colonies from the Hif- $1 \alpha$-PA-transduced MSCs. Consistent with this observation, it was shown that mice lacking Hif- $1 \alpha$ demonstrated a markedly decreased trabecular bone volume and overall reduced bone formation, ${ }^{50}$ revealing the essential role of Hif- $1 \alpha$ for self-renewing expansion of mesenchymal progenitors during development.

In contrast, the mass populations of MSCs, while exhibiting a moderate increase in proliferation in response to hypoxia, did not respond to the stabilization of Hif- $1 \alpha-\mathrm{PA}$, indicating that such moderate changes in proliferation should reflect a Hif- $1 \alpha$-independent hypoxic response of the mass population. Thus, heterogeneity exists among MSCs in their response to hypoxia, in such way that mesenchymal progenitors are selective target cell subsets responding to Hif- $1 \alpha$ stabilization. The biological significance of such selective targeting of Hif- $1 \alpha$ on progenitor population remains to be clarified. However, considering the notion that MSCs have a crucial role as a microenvironment in response to tissue damage, ${ }^{4}$ it is possible that the selective proliferation of mesenchymal progenitors by Hif- $1 \alpha$ could comprise a reactive proliferation of MSCs during hypoxic injuries of tissues. Further studies on the physiological significance of mesenchymal progenitors need to be pursued for more comprehensive understanding.

Interestingly, stabilization of Hif- $1 \alpha$ induced pluripotent genes for both MSCs expressing Hif- $\alpha$-PA or MSCs exposed to hypoxia, where the elevated levels of expression were maintained over 7 days of hypoxic period. Taking that the level of endogenously stabilized form of Hif- $1 \alpha$ is rapidly decreased by 3 days after hypoxic culture, it is possible that additional epigenetic changes participate in the alteration of gene expression in a manner that epigenetic changes coax the gene expression changes even after Hif- $1 \alpha$ is destabilized in the cells. Consistent with the possibility, we have observed changes in the CpG methylation in the Oct-4 promoters for MSCs exposed to hypoxia (data not shown). Whether these induction of pluripotent genes are specific to the mesenchymal progenitor cell populations as observed for the self-renewal of colony-forming cells remains to be determined.

Notably, while our studies, and studies of others, showed that osteogenic differentiation is inhibited with Hif- $1 \alpha$ stabilization or under hypoxic conditions, ${ }^{30,36,40}$ various observations were reported on the role of Hif- $1 \alpha$ on adipogenic differentiation. For example, the stabilization of Hif- $1 \alpha$ resulted in an increase in adipogenic markers in a pre-osteoblast cell line, ${ }^{38}$ whereas our study of MSCs and other studies using preadipocytes showed that adipogenic differentiation is inhibited under hypoxic conditions. ${ }^{37,39}$ Moreover, discrepancies were also observed for the role of Hif- $1 \alpha$ in MSCs, that is, while our studies showed a direct contribution of Hif- $1 \alpha$ for the selfrenewal of mesenchymal progenitors as well as for the inhibition of adipogenic differentiation, a study using shRNA against Hif- $1 \alpha$ did not observe the inhibition of colony formation or adipogenic differentiation. ${ }^{40}$ The reasons for these discrepancies related to the biological effects of Hif- $1 \alpha$ in MSCs with respect to the study models are currently unclear. However, considering the fact that the transcriptional milieu should be distinct with respect to cell type as well as the stages of differentiation of MSCs, it is possible that the biological targets of Hif- 1 in each stage towards terminal differentiation could become different between the transient and sustained stabilization of Hif- $1 \alpha$. Likewise, as the study by Tamama et al. ${ }^{40}$ used TERT (telomerase reverse transcriptase)immortalized MSCs, where alteration in characteristics of MSCs can occur, ${ }^{51,52}$ difference in the cell type used in the study could also have had a role. Moreover, Hif- $1 \alpha$ is largely regulated at the protein stability level with negative-feedback regulation of prolyl hydroxylase (PHD2, PHD3) by Hif- $1 \alpha$ protein itself. ${ }^{53}$ Thus it is possible that the protein stabilization of Hif- $1 \alpha$ could be subject to variation during the culture period in the model using knockdown of Hif- $1 \alpha$ at the mRNA level. Notably, endogenous level of Hif- $1 \alpha$ was relatively maintained at high level during the 2 weeks of osteogenic differentiation. Whether the protein stability of Hif- $1 \alpha$ is differentially regulated during proliferative or differentiation process remains to be determined.

Interestingly, MSCs stabilized with Hif- $1 \alpha$ expressed higher levels of neuron-specific genes, even during expansion of cultures in the absence of differentiation-inducing agents, suggesting that the cells were, to a certain level, primed toward neuron-like cells. In agreement with our observations, Pacary et al. ${ }^{54}$ showed that MSCs expressed neuronal markers and responded to neuronal transmitters in a manner that was dependent on Hif- $1 \alpha$ stabilization. At present, the biological significance of these findings remains to be elucidated. However, it is noteworthy that recent developmental studies have shown that neural crest-derived stem cells should be the potential origin of MSCs and that significant populations of MSCs share the characteristics of neural crest stem cells. ${ }^{5-57}$ Therefore, it should be an interesting possibility that observed priming of MSCs towards neuron-like cells following Hif- $1 \alpha$ stabilization could reflect their shift towards a more primitive stage of origin, that is, neural crest-like stage of the cells. However, further studies will be needed in order to further explore and confirm these possibilities.

Nevertheless, our study reveals a previously unrecognized pivotal role of Hif- $1 \alpha$ in primary BM-derived MSCs in regulating their self-renewal and primitive state through sustained stabilization model. Moreover, our study reveals a functional heterogeneity among MSC subsets with respect to their proliferative response to Hif- $1 \alpha$ stabilization, identifying the mesenchymal progenitors as a selective target population of Hif- $1 \alpha$. Further studies on the physiological significance of Hif- $1 \alpha$ stabilization during the various regenerative processes 
of the damaged tissues under hypoxic conditions should shed light on the functional impact of Hif- $1 \alpha$ in MSCs.

\section{CONFLICT OF INTEREST}

The authors declare no conflict of interest.

\section{ACKNOWLEDGEMENTS}

This study was supported by the National Research Foundation of Korea (NRF) and funded by the Ministry of Science, ICT \& Future Planning (No. 2011-0019352) and a grant of the Korea Health technology R\&D Project, Ministry of Health \& Welfare, Republic of Korea (A120262). We also thank Professor KW Kim (Seoul National University, Seoul, Korea) for his generous gift of Hif- $1 \alpha$ WT and the Hif- $1 \alpha$ Flag-p402A/p564A plasmid construct. SJH and O-JK were supported by a Grant (10172KFDA993) from the Korea Food and Drug Administration in 2013.

Author contributions: I-HP J-SS and SJH performed research, collected data and prepared the manuscript figures; K-HK performed research, collected data and designed research; $\mathrm{H}-\mathrm{KC}$ performed research and collected data; S-YW performed research; O-JK performed and designed research; and I-HO designed research, analyzed and interpreted data and wrote the manuscript.

1 Keating A. Mesenchymal stromal cells. Curr Opin Hematol 2006; 13: 419-425.

2 Pittenger MF, Mackay AM, Beck SC, Jaiswal RK, Douglas R, Mosca JD et al. Multilineage potential of adult human mesenchymal stem cells. Science 1999; 284: 143-147.

3 Prockop DJ. Marrow stromal cells as stem cells for nonhematopoietic tissues. Science 1997; 276: 71-74.

4 Caplan Al, Dennis JE. Mesenchymal stem cells as trophic mediators. J Cell Biochem 2006; 98: 1076-1084.

5 Giordano A, Galderisi U, Marino IR. From the laboratory bench to the patient's bedside: an update on clinical trials with mesenchymal stem cells. J Cell Physiol 2007; 211: 27-35.

6 Dezawa M, Hoshino M, Ide C. Treatment of neurodegenerative diseases using adult bone marrow stromal cell-derived neurons. Expert Opin Biol Ther 2005; 5: 427-435.

7 Horwitz EM, Gordon PL, Koo WK, Marx JC, Neel MD, McNall RY et al. Isolated allogeneic bone marrow-derived mesenchymal cells engraft and stimulate growth in children with osteogenesis imperfecta: implications for cell therapy of bone. Proc Natl Acad Sci USA 2002; 99: 8932-8937.

8 Jorgensen C, Gordeladze J, Noel D. Tissue engineering through autologous mesenchymal stem cells. Curr Opin Biotechnol 2004; 15: 406-410.

9 Magne D, Vinatier C, Julien M, Weiss P, Guicheux J. Mesenchymal stem cell therapy to rebuild cartilage. Trends Mol Med 2005; 11: 519-526.

10 Kim DW, Chung YJ, Kim TG, Kim YL, Oh IH. Cotransplantation of thirdparty mesenchymal stromal cells can alleviate single-donor predominance and increase engraftment from double cord transplantation. Blood 2004 103: 1941-1948.

11 Noort WA, Kruisselbrink AB, in't Anker PS, Kruger M, van Bezooijen RL, de Paus RA et al. Mesenchymal stem cells promote engraftment of human umbilical cord blood-derived $\operatorname{CD} 34(+)$ cells in NOD/SCID mice. Exp Hematol 2002; 30: 870-878.

12 Le Blanc K, Rasmusson I, Sundberg B, Götherström C, Hassan M, Uzunel $\mathrm{M}$ et al. Treatment of severe acute graft-versus-host disease with third party haploidentical mesenchymal stem cells. Lancet 2004; 363: 1439-1441.

13 Ringden O, Uzunel M, Rasmusson I, Remberger M, Sundberg B, Lönnies H et al. Mesenchymal stem cells for treatment of therapy-resistant graftversus-host disease. Transplantation 2006; 81: 1390-1397.

14 Colter DC, Sekiya I, Prockop DJ. Identification of a subpopulation of rapidly self-renewing and multipotential adult stem cells in colonies of human marrow stromal cells. Proc Natl Acad Sci USA 2001; 98: 7841-7845.

15 Kucia MHM, Wsoczynski M, Baskiewicz-Masiuk M, Zuba-Surma E, Machalinski B et al. A novel population of Oct-4+ SSEA-1 + CXCR4 +
CD34 + CD133 + Lin- CD45- very smal embryonic-like (VSEL) stem cells identified in human cord blood. Blood 2006; 108: 912a.

16 Smith JR, Pochampally R, Perry A, Hsu SC, Prockop DJ. Isolation of a highly clonogenic and multipotential subfraction of adult stem cells from bone marrow stroma. Stem Cells 2004; 22: 823-831.

17 Vacanti V, Kong E, Suzuki G, Sato K, Canty JM, Lee T. Phenotypic changes of adult porcine mesenchymal stem cells induced by prolonged passaging in culture. J Cell Physiol 2005; 205: 194-201.

18 Larson BL, Ylostalo J, Prockop DJ. Human multipotent stromal cells undergo sharp transition from division to development in culture. Stem Cells 2008; 26: 193-201.

19 Simon MC, Keith B. The role of oxygen availability in embryonic development and stem cell function. Nat Rev Mol Cell Biol 2008; 9: 285-296.

20 Semenza G. Targeting HIF-1 for cancer therapy. Nat Rev Cancer 2003; 3: 721-732.

21 Keith B, Simon M. Hypoxia-inducible factors, stem cells, and cancer. Cell 2007: 129: 465-472.

22 Wouters BG, Koritzinsky M. Hypoxia signalling through mTOR and the unfolded protein response in cancer. Nat Rev Cancer 2008; 8: 851-864

23 Kallio P, Wilson W, O'Brien S, Makino Y, Poellinger L. Regulation of the hypoxia-inducible transcription factor 1alpha by the ubiquitin-proteasome pathway. J Biol Chem 1999; 274: 6519-6525.

24 Ivan M, Kondo K, Yang H, Kim W, Valiando J, Ohh M et al. HIFalpha targeted for VHL-mediated destruction by proline hydroxylation: implications for 02 sensing. Science 2001; 292: 464-468.

25 Jaakkola P, Mole DR, Tian YM, Wilson MI, Gielbert J, Gaskell SJ et al. Targeting of HIF-alpha to the von Hippel-Lindau ubiquitylation complex by 02-regulated prolyl hydroxylation. Science 2001; 292: 468-472.

26 Masson N, Willam C, Maxwell P, Pugh C, Ratcliffe P. Independent function of two destruction domains in hypoxia-inducible factor-alpha chains activated by prolyl hydroxylation. EMBO J 2001; 20: 5197-5206.

27 Ren $\mathrm{H}$, Cao Y, Zhao Q, Li J, Zhou C, Liao L et al. Proliferation and differentiation of bone marrow stromal cells under hypoxic conditions. Biochem Biophys Res Commun 2006; 347: 12-21.

28 Rosová I, Dao M, Capoccia B, Link D, Nolta J. Hypoxic preconditioning results in increased motility and improved therapeutic potential of human mesenchymal stem cells. Stem Cells 2008; 26: 2173-2182.

29 Crisostomo P, Wang Y, Markel TA, Wang M, Lahm T, Meldrum DR. Human mesenchymal stem cells stimulated by TNF-alpha, LPS, or hypoxia produce growth factors by an NF kappa B- but not JNK-dependent mechanism. Am J Physiol Cell Physiol 2008; 294: C675-C682.

30 Fehrer C, Brunauer R, Laschober G, Unterluggauer H, Reitinger S, Kloss F et al. Reduced oxygen tension attenuates differentiation capacity of human mesenchymal stem cells and prolongs their lifespan. Aging Cell 2007; 6: 745-757.

31 Kinnaird T, Stabile E, Burnett M, Epstein S. Bone-marrow-derived cells for enhancing collateral development: mechanisms, animal data, and initial clinical experiences. Circ Res 2004; 95: 354-363.

32 Grayson W, Zhao F, Bunnell B, Ma T. Hypoxia enhances proliferation and tissue formation of human mesenchymal stem cells. Biochem Biophys Res Commun 2007; 358: 948-953

33 Lee JW, Bae SH, Jeong JW, Kim SH, Kim KW. Hypoxia-inducible factor (HIF-1)alpha: its protein stability and biological functions. Exp Mol Med 2004; 36: 1-12.

34 Zhang D, Li J, Costa M, Gao J, Huang C. JNK1 mediates degradation HIF1alpha by a VHL-independent mechanism that involves the chaperones Hsp90/Hsp70. Cancer Res 2010; 70: 813-823.

35 Flugel D, Gorlach A, Michiels C, Kietzmann T. Glycogen synthase kinase 3 phosphorylates hypoxia-inducible factor 1alpha and mediates its destabilization in a VHL-independent manner. Mol Cell Biol 2007; 27: 3253-3265.

36 D'Ippolito G, Diabira S, Howard GA, Roos BA, Schiller PC. Low oxygen tension inhibits osteogenic differentiation and enhances stemness of human MIAMI cells. Bone 2006; 39: 513-522.

37 Floyd ZE, Kilroy G, Wu X, Gimble JM. Effects of prolyl hydroxylase inhibitors on adipogenesis and hypoxia inducible factor 1 alpha levels under normoxic conditions. J Cell Biochem 2007; 101: 1545-1557.

38 Irwin R, LaPres JJ, Kinser S, McCabe LR. Prolyl-hydroxylase inhibition and HIF activation in osteoblasts promotes an adipocytic phenotype. J Cell Biochem 2007; 100: 762-772.

39 Lin Q, Lee YJ, Yun Z. Differentiation arrest by hypoxia. J Biol Chem 2006 281: 30678-30683. 
40 Tamama K, Kawasaki H, Kerpedjieva SS, Guan J, Ganju RK, Sen CK. Differential roles of hypoxia inducible factor subunits in multipotential stromal cells under hypoxic condition. J Cell Biochem 2011; 112: 804-817.

41 Kim D, Chung Y, Kim T, Kim Y, Oh I. Cotransplantation of third-party mesenchymal stromal cells can alleviate single-donor predominance and increase engraftment from double cord transplantation. Blood 2004; 103: 1941-1948.

42 Oh I, Eaves C. Overexpression of a dominant negative form of STAT3 selectively impairs hematopoietic stem cell activity. Oncogene 2002; 21: 4778-4787.

43 Oh I, Fabry ME, Humphries RK, Pawliuk R, Leboulch P, Hoffman R et al. Expression of an anti-sickling beta-globin in human erythroblasts derived from retrovirally transduced primitive normal and sickle cell disease hematopoietic cells. Exp Hematol 2004; 32: 461-469.

44 Pittenger M, Mackay AM, Beck SC, Jaiswal RK, Douglas R, Mosca JD et al. Multilineage potential of adult human mesenchymal stem cells. Science 1999; 284: 143-147.

45 Woodbury D, Schwarz E, Prockop D, Black I. Adult rat and human bone marrow stromal cells differentiate into neurons. J Neurosci Res 2000; 61: 364-370.

46 Hamill OP, Marty A, Neher E, Sakmann B, Sigworth FJ. Improved patchclamp techniques for high-resolution current recording from cells and cellfree membrane patches. Pflugers Archiv 1981; 391: 85-100.

47 Ball SG, Shuttleworth A, Kielty CM. Inhibition of platelet-derived growth factor receptor signaling regulates Oct4 and Nanog expression, cell shape, and mesenchymal stem cell potency. Stem Cells 2012; 30: 548-560.

48 Tsai CC, Su PF, Huang YF, Yew TL, Hung SC. Oct4 and Nanog directly regulate Dnmt1 to maintain self-renewal and undifferentiated state in mesenchymal stem cells. Mol Cell 2012; 47: 169-182.

49 Hong JH, Hwang ES, McManus MT, Amsterdam A, Tian Y, Kalmukova R et al. TAZ, a transcriptional modulator of mesenchymal stem cell differentiation. Science 2005; 309: 1074-1078.
50 Shomento SH, Wan C, Cao X, Faugere MC, Bouxsein ML, Clemens TL et al. Hypoxia-inducible factors 1alpha and 2alpha exert both distinct and overlapping functions in long bone development. J Cell Biochem 2010; 109: 196-204.

51 Liu TM, Ng WM, Tan HS, Vinitha D, Yang Z, Fan JB et al. Molecular basis of immortalization of human mesenchymal stem cells by combination of p53 knockdown and human telomerase reverse transcriptase overexpression. Stem Cells Dev 2013; 22: 268-278.

52 Okamoto T, Aoyama T, Nakayama T, Nakamata T, Hosaka T, Nishijo K et al. Clonal heterogeneity in differentiation potential of immortalized human mesenchymal stem cells. Biochem Biophys Res Commun 2002; 295: 354-361.

53 Marxsen JH, Stengel P, Doege K, Heikkinen P, Jokilehto T, Wagner T et al. Hypoxia-inducible factor-1 (HIF-1) promotes its degradation by induction of HIF-alpha-prolyl-4-hydroxylases. Biochem J 2004; 381: 761-767.

54 Pacary E, Legros H, Valable S, Duchatelle P, Lecocq M, Petit E et al. Synergistic effects of $\mathrm{CoCl}(2)$ and ROCK inhibition on mesenchymal stem cell differentiation into neuron-like cells. J Cell Sci 2006; 119: 26672678.

55 Bronner ME, LeDouarin NM. Development and evolution of the neural crest: an overview. Dev Biol 2012; 366: 2-9.

56 Dupin E, Sommer L. Neural crest progenitors and stem cells: from early development to adulthood. Dev Biol 2012; 366: 83-95.

57 Wislet-Gendebien S, Laudet E, Neirinckx V, Alix P, Leprince P, Glejzer A et al. Mesenchymal stem cells and neural crest stem cells from adult bone marrow: characterization of their surprising similarities and differences. Cell Mol Life Sci 2012; 69: 2593-2608.

(c) $(1) \Theta \Theta$ This work is licensed under a Creative Commons
Attribution-NonCommercial-NoDerivs 3.0 Un-
ported License. To view a copy of this license, visit http://
creativecommons.org/licenses/by-nc-nd/3.0/

Supplementary Information accompanies the paper on Experimental \& Molecular Medicine website (http://www.nature.com/emm) 